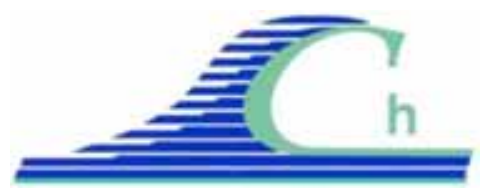

XII ${ }^{\text {èmes }}$ Journées Nationales Génie Côtier - Génie Civil

Cherbourg, 12-14 juin 2012

DOI:10.5150/jngcgc.2012.039-R @ Editions Paralia CFL

disponible en ligne - http://www.paralia.fr - available online

\title{
Modélisation physique de la liquéfaction au pied d'une structure côtière
}

\section{Valérie RAMELIARISON ${ }^{1}$, Hervé MICHALLET ${ }^{2}$, Céline BERNI ${ }^{2}$, Maxime BERGONZOLI ${ }^{2}$, Jean-Marc BARNOUD ${ }^{2}$, Eric BARTHELEMY ${ }^{2}$}

\author{
1. CETMEF/DS/LGCE, Technopôle Brest Iroise, BP5, 29280 Plouzané, France. \\ valerie.rameliarison@developpement-durable.gouv.fr \\ 2. LEGI (UMR 5519 CNRS, G-INP, UJF), BP53, 38041 Grenoble cedex, France.
}

\section{Résumé :}

Des expériences sont réalisées dans un canal à houle de $11 \mathrm{~m}$ de long et de $55 \mathrm{~cm}$ de large dans le but de modéliser la liquéfaction du lit sédimentaire au pied d’une structure côtière sous l'action du déferlement des vagues. Une paroi transversale est insérée dans le canal dont le fond est constitué de sédiments légers (densité 1,19). Les pressions interstitielles sont mesurées au niveau de la paroi et à différentes profondeurs. Plusieurs séquences de vagues sont générées, pour différents états initiaux du sol, plus ou moins lâche et plus ou moins saturé. Le seuil de liquéfaction est atteint juste avant l'impact de la vague sur la paroi et au retrait de la vague. Le phénomène est d'autant plus net que le sol initial est lâche et non saturé ; des mouvements de forte amplitude sont alors observés ainsi que l'échappement de bulles d'air du lit sédimentaire. Au fil des essais les sédiments sont de moins en moins mobilisés, la liquéfaction n'apparaît que très brièvement et le sol tend vers un état compact et saturé.

\section{Mots clés :}

Liquéfaction - Erosion - Pressions interstitielles - Vague - Mouvements sédimentaires

\section{Introduction}

La sollicitation de la houle combinée au recul du trait de côte fragilise et déstabilise les structures côtières et portuaires. Les couplages entre l'écoulement dynamique et les caractéristiques du lit sédimentaire au voisinage des ouvrages peuvent entrainer des phénomènes de liquéfaction du sol qui facilitent son érosion (SUMER et al., 2001 ; DE GROOT et al., 2006). Une expérience de terrain réalisée à Capbreton (MORY et al., 2007) a mis en évidence le rôle de la teneur en gaz sur la transmission des pressions interstitielles et donc sur la liquéfaction du lit. Les résultats sont conformes à la théorie de SAKAI et al. (1992). L'objectif de cette étude est de reproduire en laboratoire et d'observer le comportement du lit lors du déferlement sur une structure verticale.

\section{Matériel et méthodes}

Le modèle physique est réalisé dans le canal à houle du Laboratoire des Ecoulements Géophysiques et Industriels. Ce canal de section rectangulaire mesure $36 \mathrm{~m}$ de long, 
$55 \mathrm{~cm}$ de large et 1,3 $\mathrm{m}$ de haut. Ses parois verticales sont en verre afin d'assurer le suivi visuel des expériences. Pour ce modèle, seule une portion du canal est utilisée, entre 0 et $11 \mathrm{~m}$ (voir figure 1). La première extrémité est un batteur de type piston générateur de houle. La coupure à $x=11 \mathrm{~m}$ est matérialisée par une paroi en plexiglas aux dimensions intérieures du canal et modélisant la présence d'une structure côtière. Cette paroi est percée en huit points alignés verticalement sur une partie de sa hauteur pour permettre l'installation de capteurs de pression. Au pied de la paroi, une plage de pente 1:20 est construite entre les abscisses $x=2 \mathrm{~m}$ et $x=11 \mathrm{~m}$. Elle est constituée d'un sédiment plastique (PMMA) de faible masse volumique $\left(1,19 \mathrm{~g} / \mathrm{cm}^{3}\right)$ et de diamètre médian $d_{50}=0,6 \mathrm{~mm}$ (GRASSO et al., 2009). Les caractéristiques de ce sédiment ont été choisies de façon à faciliter l'apparition de la liquéfaction, en meilleur accord avec le milieu naturel par rapport aux expériences en modèle réduit existantes (TZANG \& OU, 2006). L'échelle de longueur est d'environ $1 / 10$ par rapport aux conditions de vague en milieu naturel. La profondeur d'eau est fixée à $55,3 \mathrm{~cm}$ au niveau du batteur, elle est d'environ $17 \mathrm{~cm}$ au niveau du mur.

Douze sondes capacitives sont positionnées le long du profil, permettant de mesurer la dénivellation de la surface libre sous l'effet des vagues. Une sonde à ultrasons se déplaçant sur des rails au dessus du canal permet de relever la bathymétrie. Quatre capteurs de pressions fixés à la paroi transversale mesurent soit la pression dans la colonne d'eau soit les pressions interstitielles dans le lit sédimentaire (figure 1).

Les expériences consistent à générer une onde par un mouvement de recul puis d'avancée du batteur (demi-sinusoïde de durée 2 s) qui produit une vague principale de hauteur $10 \mathrm{~cm}$ environ, suivie d'une deuxième plus petite (voir figure 2a). Les vagues sont précédées d'une onde de dépression. La première vague se brise exactement sur la paroi à $t=6 \mathrm{~s}$, alors que la deuxième ne déferle pas. Durant chaque essai les pressions interstitielles sont mesurées au niveau de la paroi.

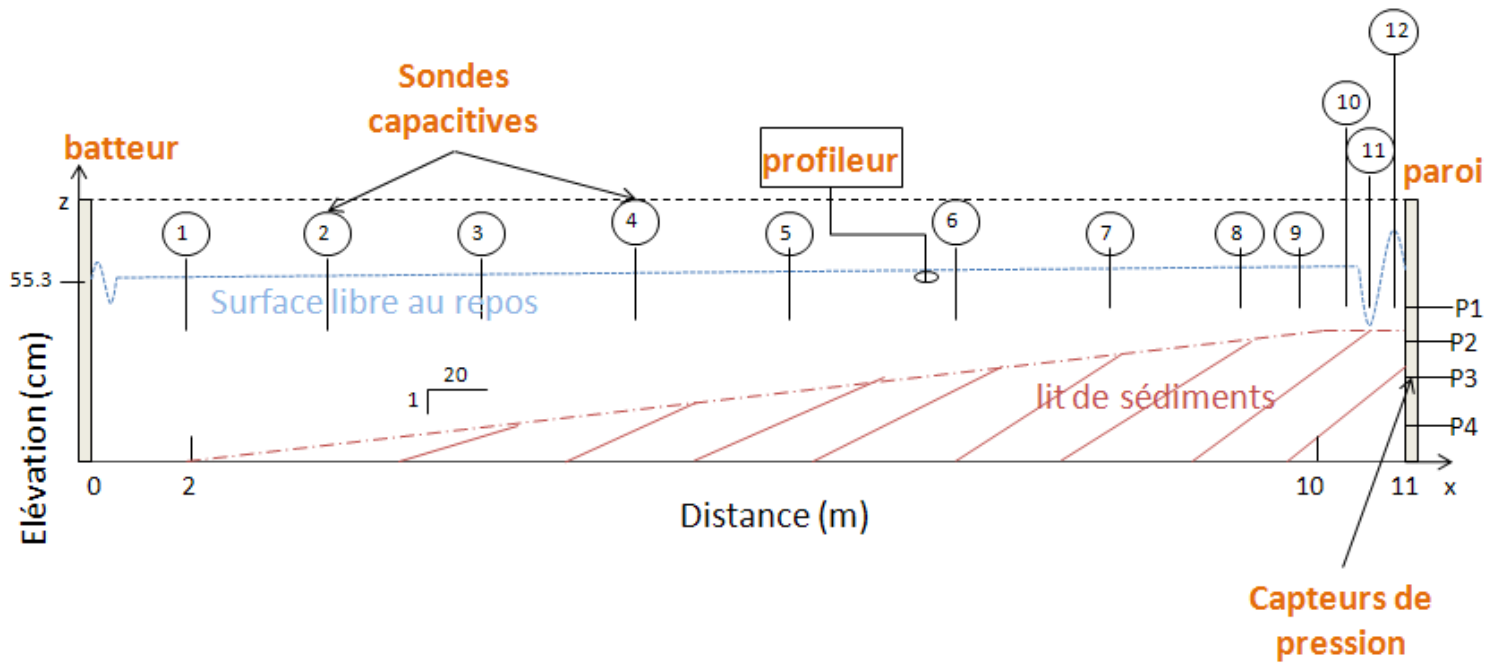

Figure 1. Schéma du dispositif expérimental. 


\section{XII ${ }^{\text {èmes }}$ Journées Nationales Génie Côtier - Génie Civil \\ Cherbourg, 12-14 juin 2012}

La nature du lit initial est déterminée préalablement au premier essai ; elle n’est plus (manuellement) retouchée par la suite. Un cycle de vidange et de remplissage du canal permet de piéger des bulles d'air à l'intérieur du sédiment (lit non saturé). Le remaniement manuel du haut de plage permet d'obtenir un lit plus lâche. La porosité du lit est estimée entre 0,43 (compact) et 0,52 (lâche). La séquence complète est répétée 6 fois pour 4 états de sol initiaux différents : compact saturé, compact non saturé, lâche saturé et lâche non saturé. Après stabilisation de la surface libre, chaque essai est ponctué par un relevé bathymétrique effectué au milieu du canal.

Les mouvements sédimentaires au pied de la structure sont filmés en vue de côté à travers la paroi en verre du canal. Une caméra DV de résolution $720 \times 576$ pixels est utilisée. Les images extraites de ces vidéos (25 images par seconde, 0,6 mm par pixel) sont traitées grâce à un logiciel de PIV (Davis). Le calcul des vecteurs de déplacement est exécuté entre deux images consécutives, sur des mailles de $16 \times 16$ pixels. Seuls les mouvements dans le sol sont analysés.

\section{Mesures de pressions interstitielles}

Les signaux de pression présentés sur la figure $2 b$ sont relevés au moment de l'impact de la vague sur la paroi, pour un sol partiellement saturé. Le capteur P1 est situé dans la colonne d'eau au niveau du lit, les trois autres sont dans le lit sédimentaire, espacés de $d=4 \mathrm{~cm}$. La série temporelle des différences de pression entre deux capteurs consécutifs est présentée sur la figure 2c. Le seuil de liquéfaction, (à la porosité estimée, voir MORY et al., 2007), est indiqué en pointillés. Ce seuil définit la différence de pression au sein d'une couche de sol d'épaisseur $d$ nécessaire à sa liquéfaction et correspond à son poids apparent.

Avant l'arrivée de la vague, la pression dans le sol chute. Cette tendance est particulièrement marquée dans les couches supérieures. Cela conduit à atteindre le seuil de liquéfaction. A l'impact proprement dit ( $t=6 \mathrm{~s})$, des fluctuations très haute fréquence sont enregistrées puis la pression devient brutalement plus forte dans les couches supérieures. Le phénomène s'inverse à nouveau à la descente de la vague et conduit à nouveau au seuil de liquéfaction ( $t \sim 7 \mathrm{~s}$ ). Cette même suite de phénomènes se reproduit de façon atténuée pour la deuxième vague de faible amplitude qui se réfléchit contre le mur sans déferler ( $t \sim 8$ à $9 \mathrm{~s}$ ). La pression décroît ensuite lentement vers la pression hydrostatique, ce qui correspond à la sédimentation du sol liquéfié.

Dans le même temps, des bulles d'air s'échappent du lit sédimentaire, comme le montrent les enregistrements vidéo. Au fil des essais, les fluctuations des différences de pression interstitielle s'atténuent, à mesure que le sol tend vers un état compact et saturé.

\section{Observation des mouvements sédimentaires}

Les mouvements du lit sédimentaire sont observés parallèlement à l'évolution des signaux de pression. Seule la partie la plus mobile est représentée, soit environ les 11 
premiers centimètres de sol. Les déplacements sont calculés et représentés en quatre instants repérés temporellement sur la figure 3. De plus, le module du tenseur des contraintes est estimé, ainsi que le rotationnel et la divergence de la vitesse. Ces grandeurs sont choisies comme estimateurs des zones de fort cisaillement, en compaction (pour des valeurs négatives de la divergence) ou en dilatation (divU $>0$ ). Les quatre instants choisis correspondent à la phase de dépression à l'avant de la vague, à l'impact, à l'instant où la vague se brise et enfin au retrait de la vague.

Dans la phase précédant l'impact ( $\mathrm{t}=5,7 \mathrm{~s}$, cf. figure 3), la dépression plus forte dans la couche supérieure de sol est associée à un écoulement vers le mur et vers le haut. C'est la première phase de liquéfaction du sol, caractérisée par des valeurs globalement positives de la divergence de la vitesse. Les sédiments sont soulevés, commencent à perdre contact et à se mêler à la phase liquide, ce qui explique la surpression enregistrée en profondeur (figure 2b).
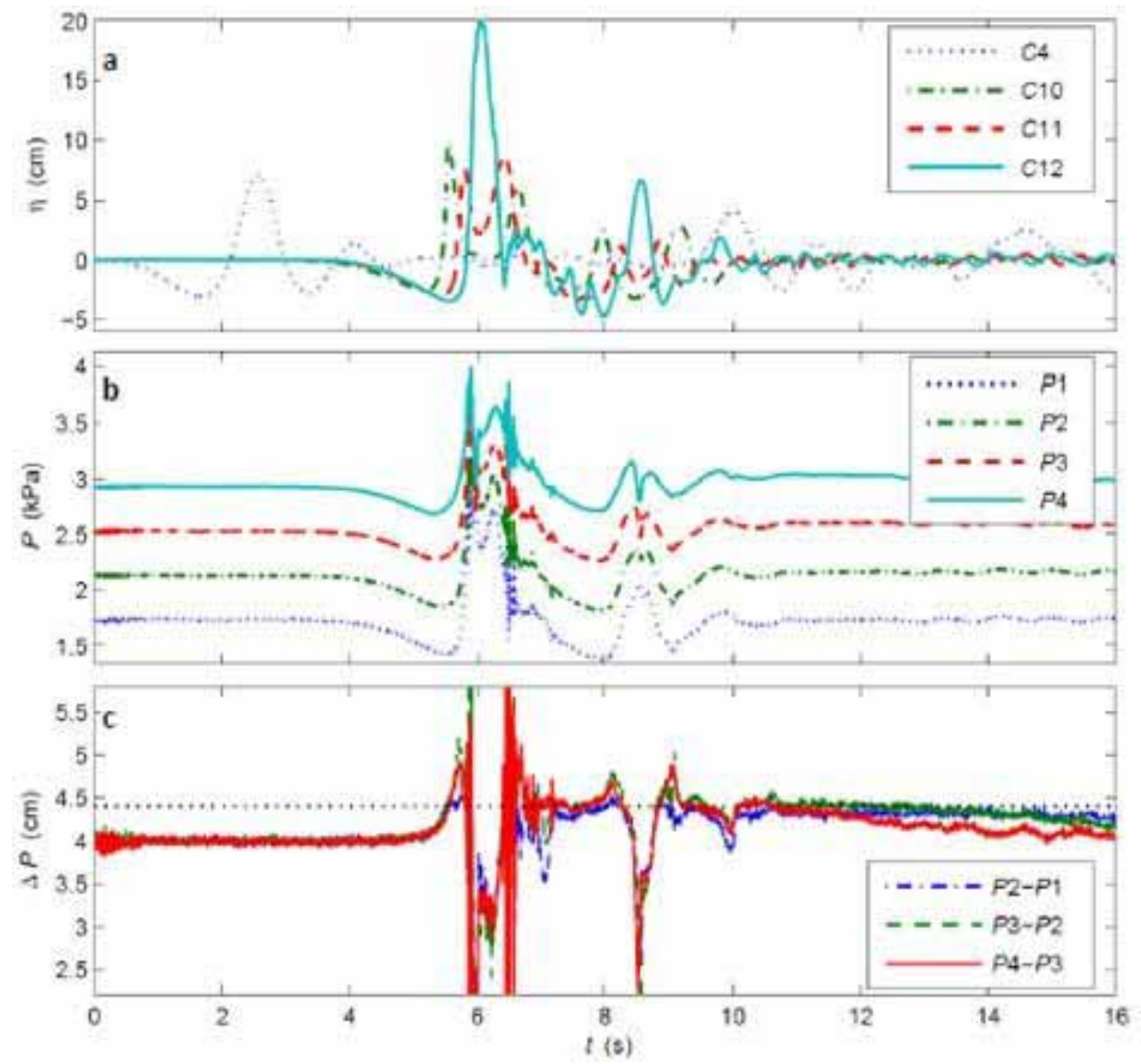

Figure 2. Série temporelle : du niveau de surface libre en $x=5 m(C 4), x=10,3 m$ (C10), $x=10,7 \mathrm{~m}$ (C11) et $x=11 \mathrm{~m}$ (C12) (a); des pressions mesurées (en $\mathrm{kPa}$ ) contre le mur

au niveau du lit (P1) et tous les $4 \mathrm{~cm}$ jusqu'à $12 \mathrm{~cm}$ de profondeur dans le sol (b) ; différences de pression (en équivalent hauteur d'eau) entre deux capteurs (c), le seuil de liquéfaction (MORY et al.,2007) est indiqué en pointillés. 


\section{XII ${ }^{\text {èmes }}$ Journées Nationales Génie Côtier - Génie Civil \\ Cherbourg, 12-14 juin 2012}
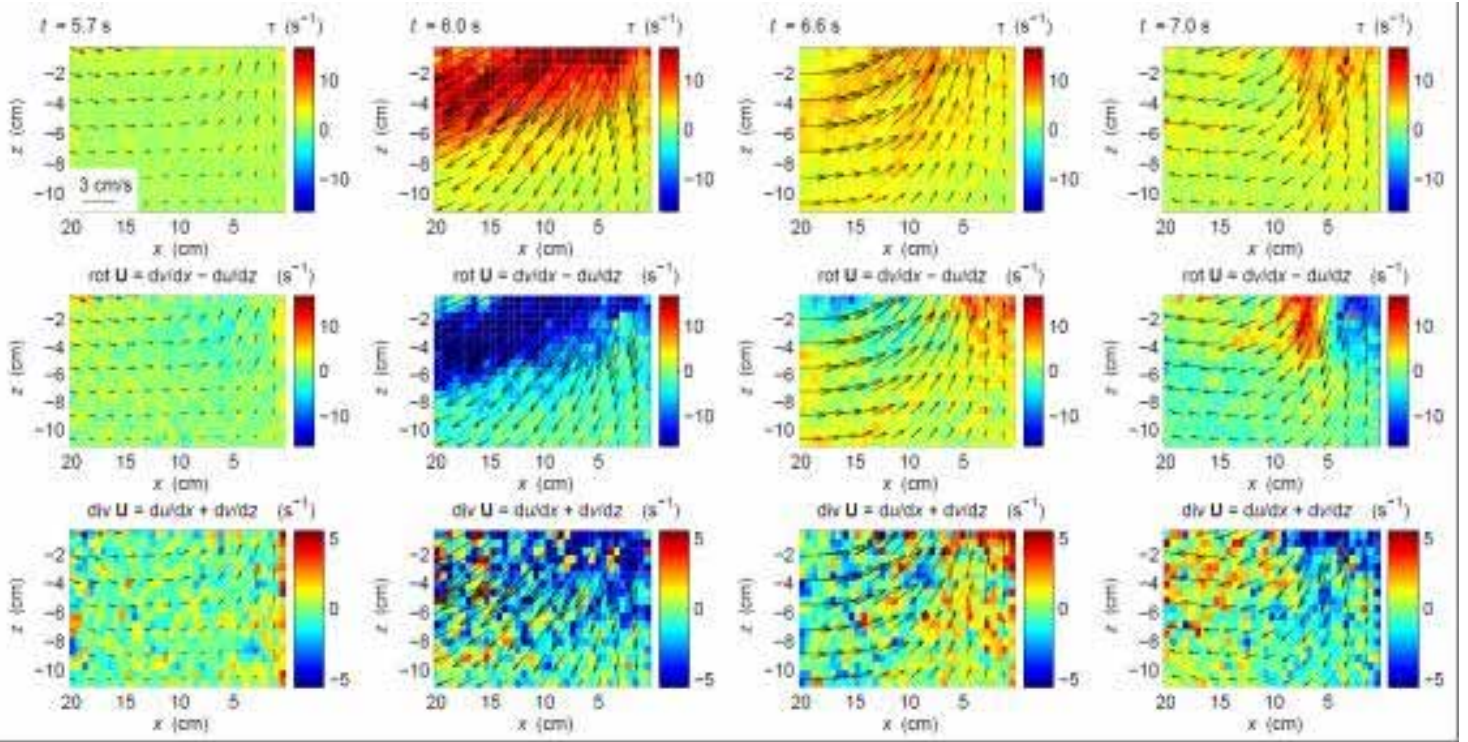

Figure 3. Champs de vitesse des mouvements sédimentaires à quatre instants de la série temporelle de la figure 2, superposés à l'estimation du cisaillement (en haut), du rotationnel (au milieu) et de la divergence (en bas) de la vitesse. La paroi verticale est en $x=0$, la position du lit en $\mathrm{z}=0$.

A l'impact ( $\mathrm{t}=6 \mathrm{~s}$ ), l'écoulement est orienté vers le bas et vers le large. Les mouvements sédimentaires sont beaucoup plus importants que lors de la phase précédente. Cela correspond à un mouvement global d'une portion de sol, ainsi que le montre la zone de fort cisaillement et fort rotationnel. Les valeurs de divergence traduisent une compaction importante du sol. Lorsque la vague se brise $(t=6,6 \mathrm{~s})$ et se retire $(t=7 \mathrm{~s})$, le cycle de dilatation/compaction se reproduit, comme le montrent les deux colonnes de droite de la figure 3. La série temporelle des différences de pression est tracée sur la figure 4, en regard des moyennes sur la zone considérée $(11 \mathrm{~cm}$ de profondeur et pour les $20 \mathrm{~cm}$ les plus proches du mur) du cisaillement, du rotationnel et de la divergence. Dans le cas de la divergence, la moyenne sur une zone très proche du mur est aussi calculée. Les instants de fort cisaillement et fort rotationnel correspondent clairement à l'impact et au retrait de la vague. La deuxième vague produit une signature similaire (à t 8.5 à 9 s) par rapport à la signature de la vague principale. Les valeurs de la moyenne de la divergence dans la zone proche du mur indiquent nettement les phases de dilatation et de compaction, en bon accord avec les variations de pression enregistrées.

\section{Evolution au fil des essais}

$\mathrm{Au}$ fur et à mesure des essais les sédiments sont de moins en moins mobilisés. L'ensemble de ces mouvements conduit à un tassement progressif du lit sédimentaire au pied de la paroi (sur une distance d'environ 1,5 m). Une érosion d'environ $4 \mathrm{~cm}$ est mesurée pour le lit initialement lâche et non saturé (figure 5a). 

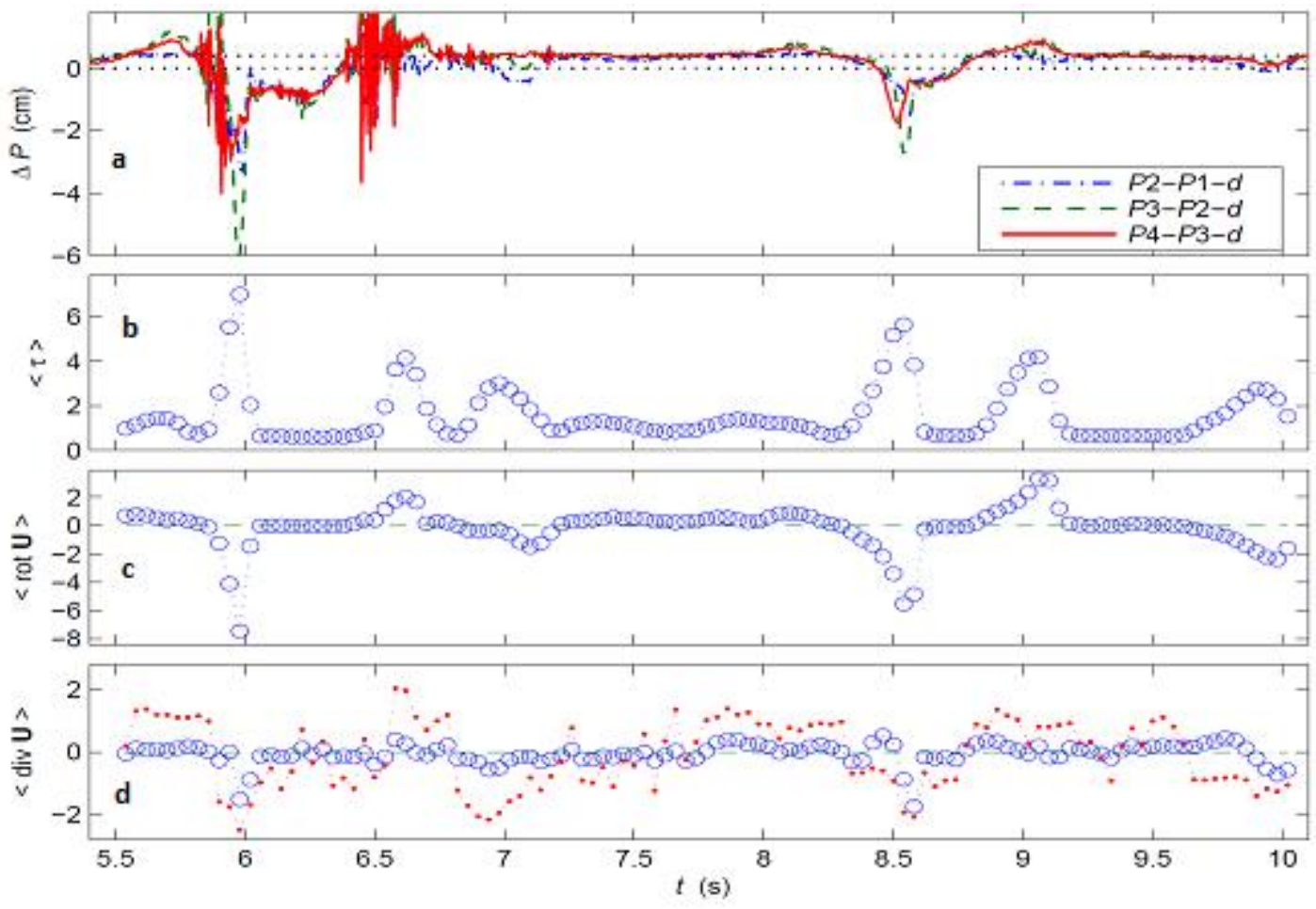

Figure 4. Série temporelle des différences de pression (la pression hydrostatique, $d=4 \mathrm{~cm}$, est retranchée) contre le mur (a) et des moyennes du cisaillement (b), $d u$ rotationnel (c) et de la divergence (d). Les moyennes sont calculées sur l'ensemble du champ PIV représenté figure 3 (o) et sur les 3 derniers cm proches du mur (.) pour la divergence.

Dans le cas initialement compact et saturé, on constate un important transport sédimentaire sur les deux derniers mètres du profil (figure 5b). Des zones d'érosion et d'accrétion se forment. Au pied de la paroi, le lit est érodé sur $2 \mathrm{~cm}$.

L'évolution des profils pour les quatre états initiaux de lit permet de mettre en évidence une compaction du lit sédimentaire d'autant plus importante que le lit est initialement lâche et non saturé (figure 6). La perte de volume sédimentaire est calculée entre 9,5 et $11 \mathrm{~m}$ et sur toute la hauteur du profil. Dans le cas initialement compact et saturé le volume sédimentaire n’évolue quasiment pas, ce qui est cohérent avec le transport sédimentaire observé sur la figure $5 \mathrm{~b}$. Au regard des pertes de volume calculées pour les trois autres états de lit (entre 1 et 5\%), le tassement correspond à la perte de porosité du lit (écart estimé de 9\% entre les états lâche et compact, voir §2). On peut remarquer que les quatre états finaux ne sont pas exactement les mêmes ; il semble que la compaction soit plus efficace à partir d'un lit non saturé. Enfin, il est intéressant de noter la complémentarité des pertes volumiques entre les états lâche saturé et compact non saturé. Pour l'état lâche saturé le tassement correspond à un réarrangement des grains 


\section{XII ${ }^{\text {èmes }}$ Journées Nationales Génie Côtier - Génie Civil \\ Cherbourg, 12-14 juin 2012}

d'un état lâche vers un état compact ; pour l'état compact non saturé, il correspond plus à une perte de teneur en air (échappement de bulles d'air). La somme de ces pertes volumiques (Lâche Saturé: -1.5\%, Compact Non Saturé: -2.9\%) se retrouve bien pour le tassement du lit lâche non saturé (-4,4\%).
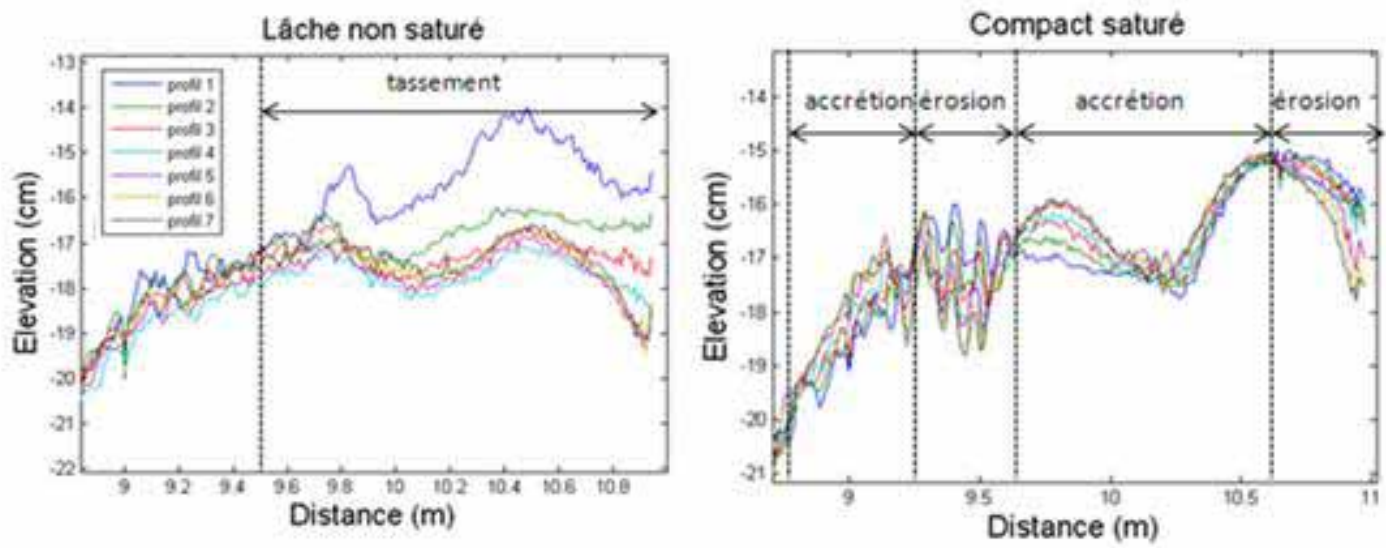

Figure 5. Evolution de la bathymétrie au fil des essais pour un cas de lit initialement lâche et non saturé (a) ou compact saturé (b). Le profil 1 correspond à la bathymétrie avant les essais. L'élévation nulle est la position de la surface libre au repos.

\section{Conclusions}

Nous avons proposé dans cet article un modèle physique en canal de la liquéfaction au pied d'une structure côtière sous l'effet des vagues. Le protocole expérimental mis en œuvre nous assure la reproductibilité des essais en canal ainsi que la différenciation de plusieurs états de lit initiaux saturé ou non saturé et lâche ou compact. Les phénomènes observés (évolution des pressions interstitielles, liquéfaction) sont semblables à ceux mis en évidence lors de la campagne de terrain de Capbreton, notamment la chute de pression dans le lit sédimentaire à l'impact de la vague (BONJEAN et al., 2004). Cette étude en canal complète ces expériences de terrain en mettant en avant les conditions de liquéfaction du lit. L'analyse PIV nous a par ailleurs permis de suivre de plus près les mouvements sédimentaires et de les mettre en parallèle avec l'évolution des pressions interstitielles. Le lit est inégalement mobilisé (vitesses, cisaillement). Un lit initialement lâche et non saturé est plus facilement liquéfié et érodé ; il se tasse à mesure que les bulles d'air s'échappent et devient plus compact et saturé. La quantification précise de la teneur en air du lit est encore à l'étude.

Remerciements : Cette étude a été réalisée dans le cadre du projet Hydro-Fond (C2D2/RGCU, MEDDTL). Le support technique de L. Vignal, M. Lagauzère et F. Bonnel a été très fortement apprécié, ainsi que les conseils de J. Chauchat pour l'estimation du module de cisaillement. 


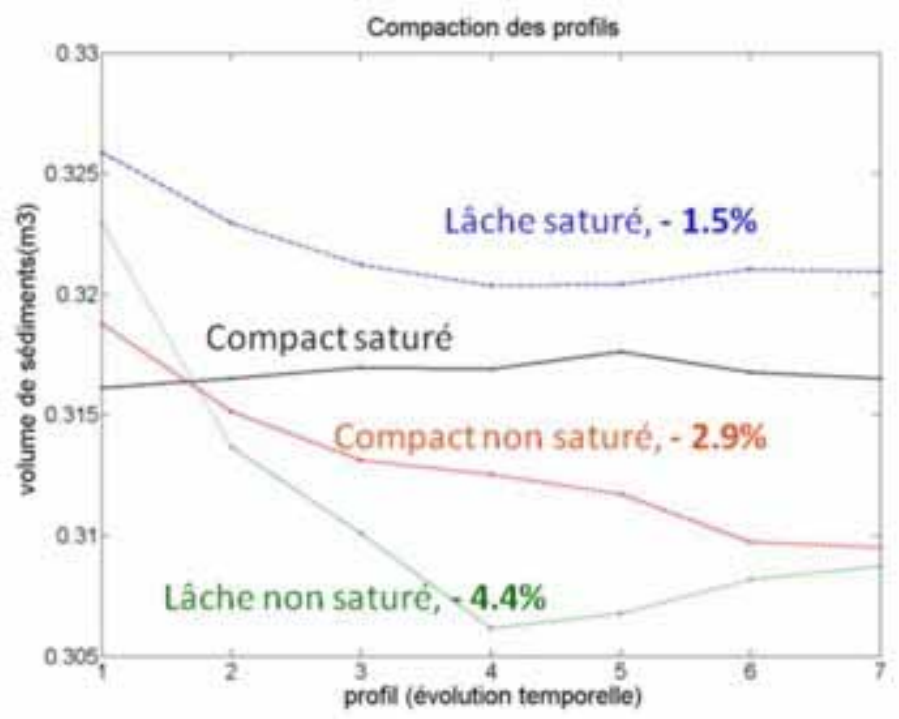

Figure 6. Evolution des volumes sédimentaires (entre 9,5 et $11 \mathrm{~m}$ ) au fil des essais.

\section{Références bibliographiques}

BONJEAN D., FORAY P., PIEDRA-CUEVA I., MICHALLET H., BREUL P., HADDANI Y., MORY M., ABADIE S. (2004). Monitoring of the foundations of a coastal structure submitted to breaking waves : occurrence of momentary liquefaction. Proc. 14th ISOPE, vol.2, pp 585-592.

DE GROOT M.B., BOLTON M.D., FORAY P., MEIJERS P., PALMER A.C., SAWICKI A., TEH T.C. (2006). Physics of liquefaction phenomena around marine structures. Journal of Waterway, Port, Coastal and Ocean Engineering, Vol. 132 (4), pp 227-243. doi:10.1061/(ASCE)0733-950X(2006)132:4(227)

GRASSO F., MICHALLET H., BARTHELEMY E., CERTAIN R. (2009). Physical modeling of intermediate cross-shore beach morphology: Transients and equilibrium states. J. Geophys. Res., 114, C09001. doi:10.1029/2009JC005308

MORY M., MICHALLET H., BONJEAN D., PIEDRA-CUEVA I., BARNOUD J.-M., FORAY P., ABADIE S., BREUL P. (2007). A field study of momentary liquefaction caused by waves around a coastal structure. Journal of Waterway, Port, Coastal and Ocean Engineering, Vol. 133(1), pp 28-38. doi:10.1061/(ASCE)0733-950X(2007)133:1(28)

SAKAI T., HATANAKA K., MASE H. (1992). Wave-Induced Effective Stress in Seabed and Its Momentary Liquefaction. Journal of Waterway, Port, Coastal and Ocean Engineering, Vol. 118, No. 2, pp 202-206. doi:10.1061/(ASCE)0733-950X(1992)118:2(202)

SUMER B.M., WHITEHOUSE R.J.S., TORUM A. (2001). Scour around coastal structures: a summary of recent research. Coastal Engineering, Vol. 44, pp 153-190. doi:10.1016/S0378-3839(01)00024-2

TZANG S.-Y., OU S.-H. (2006). Laboratory flume studies on monochromatic wavefine sandy bed interactions: Part 1. Soil fluidization. Coastal Engineering, Vol. 53(11), pp 965-982. doi:10.1016/j.coastaleng.2006.06.003 